\title{
Responses of Macaque Perirhinal Neurons during and after Visual Stimulus Association Learning
}

\author{
Cynthia A. Erickson and Robert Desimone \\ Laboratory of Neuropsychology, National Institute of Mental Health, National Institutes of Health, Bethesda, \\ Maryland 20892-4415
}

Recent lesion studies have implicated the perirhinal cortex in learning that two objects are associated, i.e., visual association learning. In this experiment we tested whether neuronal responses to associated stimuli in perirhinal cortex are altered over the course of learning. Neurons were recorded from monkeys during performance of a visual discrimination task in which a predictor stimulus was followed, after a delay, by a GO or NO-GO choice stimulus. Association learning had two major influences on neuronal responses. First, responses to frequently paired predictor-choice stimuli were more similar to one another than was the case with infrequently paired stimuli.
Second, the magnitude of activity during the delay was correlated with the magnitude of responses to both the predictor and choice stimuli. Both of these learning effects were found only for stimulus pairs that had been associated on at least $2 \mathrm{~d}$ of training. Early in training, the delay activity was correlated only with the response to the predictor stimuli. Thus, with long-term training, perirhinal neurons tend to link the representations of temporally associated stimuli.

Key words: single unit; electrophysiology; Macaca mulatta; perirhinal cortex; inferior temporal cortex; paired associates; association; learning; memory; primates; medial temporal lobe
Anatomical, behavioral, and neurophysiological studies indicate that the perirhinal portion of the inferior temporal cortex in primates plays an important role in visual memory. Perirhinal cortex receives substantial input from visual area TE (Suzuki and Amaral, 1994), a high-order visual-processing area, and projects via entorhinal cortex into the hippocampal formation. Animals with selective lesions of perirhinal cortex are impaired on tests of recognition memory, such as the delayed matching to sample (DMS) (Meunier et al., 1993) or nonmatching to sample tasks (Murray and Mishkin, 1986; Zola-Morgan et al., 1989, 1993; Meunier et al., 1993; Suzuki et al., 1993; Gaffan, 1994). Many perirhinal neurons exhibit mnemonic properties in such tasks (Eskandar et al., 1992; Fahy et al., 1993; Li et al., 1993; Miller et al., 1993; Sobotka and Ringo, 1993, 1996; Lueschow et al., 1994). Specifically, for some cells the response to a repeated visual stimulus is suppressed if it matches a previously viewed stimulus. Such suppression appears to occur automatically for any stimulus repetition, regardless of behavioral relevance, and serves to distinguish novel stimuli from familiar ones. For other perirhinal cells, the response to the choice stimulus is enhanced if it matches the sample, and in contrast to suppression, enhancement appears to depend on active working memory for the sample (Miller and Desimone, 1994). Many perirhinal neurons also respond differentially during the period immediately after stimulus presentation (Fuster and Jervey, 1981, 1982; Miller et al., 1993). Together, the heightened delay activity and the enhanced response to the sought-for choice stimulus have suggested that some perirhinal

Received Jan. 11, 1999; revised Sept. 2, 1999; accepted Sept. 3, 1999.

This work was supported by the IRP National Institute of Mental Health. We thank Drs. Earl Miller, Bharathi Jagadeesh, and Martin Paré for helpful suggestions on task design and data analysis. Barbara K. Changizi, Amy C. Durham, and Sudabeh Shirazi helped with technical aspects of the experiment.

Correspondence should be addressed to Dr. Robert Desimone, Laboratory of Neuropsychology, Building 49, Room 1B80, National Institute of Mental Health, Bethesda, MD 20892-4415. E-mail: bobd@In.nimh.nih.gov.

Copyright (C) 1999 Society for Neuroscience 0270-6474/99/1910404-13\$05.00/0 neurons are sensitized, or primed, to respond to stimuli held actively in working memory.

In addition to a role in recognition memory, perirhinal cortex may also be involved in associative memory, i.e., establishing linkages between different stimuli that have some meaningful connection. Combined lesions of the perirhinal and neighboring entorhinal cortex result in profound deficits in the learning of, and memory for, stimulus associations in monkeys (Murray et al., 1993). To test the role of perirhinal neurons in associative memory, Sakai and Miyashita (1991) recorded from perirhinal neurons in a paired-associate task. In their task, the monkey was shown a sample stimulus followed, after a delay, by a choice stimulus and was rewarded for indicating whether the choice stimulus was the correct paired associate of the sample. They reported that some perirhinal cells had delay activity that was selective for the expected choice stimulus after a given sample and that some cells responded preferentially to specific samplechoice pairs. Because all training took place before recording, it was not possible to examine the time course of any response changes relative to the association learning. In contrast to the association effects reported by Sakai and Miyashita, two other groups (Sobotka and Ringo, 1993; Gochin et al., 1994), using two different association tasks, failed to find evidence of inferior temporal participation in association learning.

To reconcile these conflicting reports and to test further how perirhinal neurons might contribute to associative memory, we developed a task in which the monkey could form associative memories of multiple pairs of stimuli over the course of a single recording session. Perirhinal neuronal responses were recorded during performance of the task using either novel (learned within a single session) or familiar (stimulus pairs with which the monkey had been trained in one or more previous sessions) stimuli. This task provided us with the opportunity to examine two aspects of association learning, namely, changes in the responses 
A

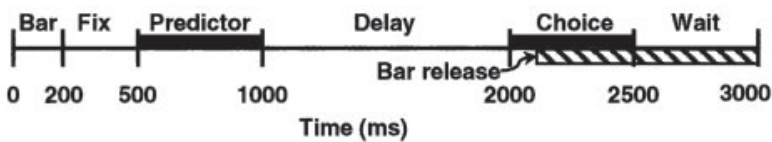

B Valid Trials (85\%)
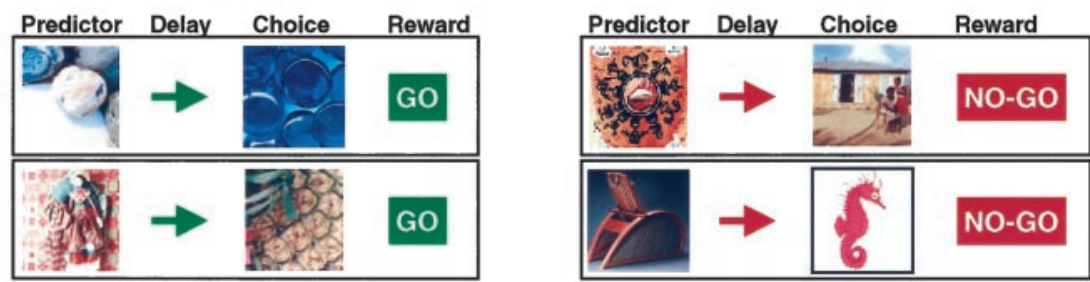

C Invalid Trials (10\%)
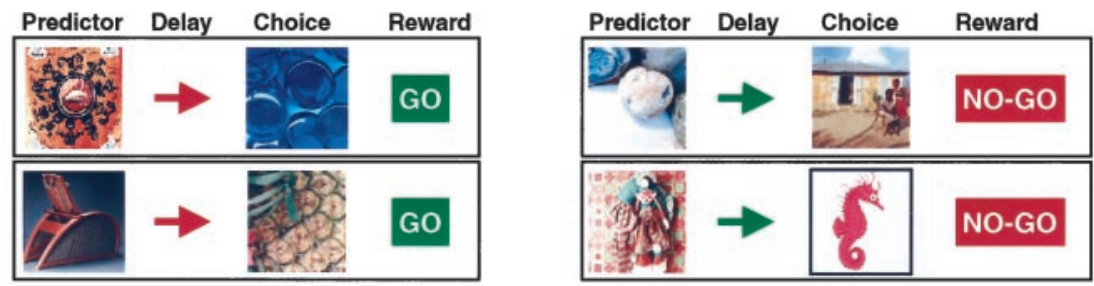

D Neutral Trials (5\%)
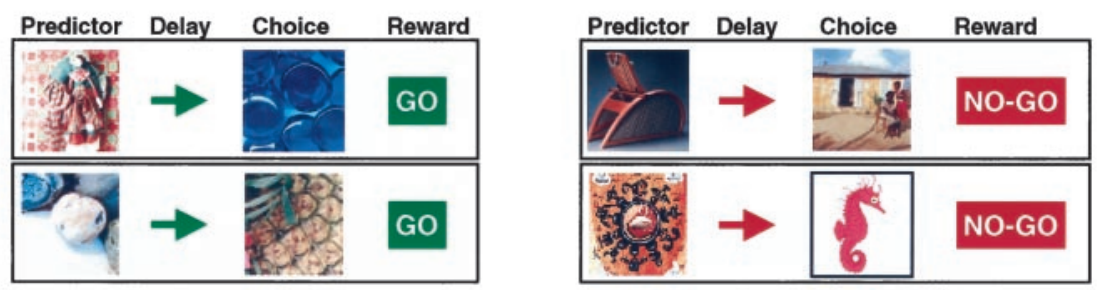

Figure 1. Representation of the task. A, The timing of stimulus presentations and the behavioral response interval in GO trials are shown. Trials were initiated by the monkey holding a bar (Bar). A fixation spot (Fix) was then presented in the center of the screen. After a random delay (average of $300 \mathrm{msec}$ ), a predictor stimulus (Predictor) was presented for $500 \mathrm{msec}$, followed by a delay (Delay) of $1000 \mathrm{msec}$. Finally, a choice stimulus (Choice) was presented. In GO trials, the monkey was rewarded for releasing the bar either during the choice stimulus presentation or within the $500 \mathrm{msec}$ after the choice stimulus was turned off (indicated by the striped horizontal bar). In NO-GO trials, the monkey was rewarded for holding the bar through the same period. Gray horizontal bars indicate when stimuli were on. $B-D$, The three types of stimulus pairings were valid $(B)$, invalid $(C)$, and neutral $(D)$, and all the stimuli in a session were used in all three types of pairings. The stimuli shown are representative of the stimuli used in the recordings. of cells to the paired stimuli and differential delay activity after a sample stimulus and before its paired choice stimulus.

\section{MATERIALS AND METHODS}

\section{Subjects}

The two adult male, 7-9 kg, rhesus monkeys (Macaca mulatta) used in this experiment were cared for according to National Institutes of Health guidelines.

\section{Stimuli}

The stimuli were $1-3^{\circ}$ square-shaped, colored pictures presented on a computer monitor. The pictures were cropped and modified from digitized photographs, artwork, or diagrams. Some of the images were clearly recognizable objects, whereas others were abstract designs or patterns. For each training set, 16 stimuli were randomly chosen from a stimulus pool of over 1000 images and were randomly paired to form eight paired associates (stimulus pairs).

Stimuli were defined as novel if the monkey had never seen them before the start of recording. In these recording sessions, the monkey's first experience with an individual stimulus was the first trial of the recording session. Stimuli were defined as familiar if the monkey had successfully learned the stimuli on at least 1 previous day. Examples of the types of stimuli used are shown in Figure 1. After a stimulus set had been established, those stimuli were not used in a different stimulus set.

\section{Behavioral task}

Task rationale. Traditional paired-associate association-learning tasks in primates are typically based on conditional performance rules, such as if A is followed by $\mathrm{C}$, press the bar, if $\mathrm{B}$ is followed by $\mathrm{C}$, do not press the bar, if A is followed by D, press the bar, if B is followed by $\mathrm{D}$, do not press the bar, etc. (Gochin et al., 1994). In some conditional designs, two choice stimuli are presented simultaneously, such as if A is followed by B and C, choose B, if D is followed by B and C, choose C, etc. (Sakai and Miyashita, 1991). Conditional designs such as these often require lengthy training on specific pairs of stimuli, and there is often no obligatory temporal relationship between the first and second stimulus in the pair; e.g., stimulus A may be followed equally often by B or C.

To examine how perirhinal neurons might contribute to associative memories acquired over both short (within a day) and long (multiple days) periods of exposure to stimuli, we developed a task in which the monkey could form associative memories of multiple pairs of stimuli over the course of a single recording session. The two previous studies that failed to find associative effects (see introductory remarks) influenced our experiment design. First, we hypothesized that neurons that respond selectively to associated stimuli were not found in the Gochin et al. (1994) experiment because a given sample stimulus was paired an equal number of times with two different choice stimuli, and both types of pairings were behaviorally relevant. Second, in the Sobotka and Ringo (1993) experiment, the associated stimuli were presented together simultaneously, and the task did not require that the animals perceive the stimulus pairs as two separate stimuli. The paired stimuli might have been perceived as a single compound stimulus. These results suggested that it might be important to maintain consistent pairings between the associated stimuli and that the stimuli be perceived as separate objects. We therefore used a paradigm based on discrimination learning, a task in which monkeys can learn new stimuli quickly after acquiring the discrimination rule. In some sessions, the monkeys learned eight new discriminanda, or choice stimuli, concurrently (termed novel stimuli). In other sessions they were tested with eight previously learned stimuli (termed familiar stimuli). Each of the eight choice stimuli was preceded by a specific predictor stimulus. Each predictor stimulus predicted the occurrence of its paired choice stimulus with $80-90 \%$ probability ("valid trials"). Thus, there were eight pairs of predictor-choice stimuli or 16 stimuli in total per session. No awareness of the stimulus-stimulus relationship was necessary for successful completion of a trial, but the monkeys could potentially respond more quickly if they learned the specific predictor-choice relationships.

When a predictor stimulus was followed by the expected choice stimulus, it was termed a valid trial. In "probe trials," a given predictor 
stimulus was followed by an unexpected choice stimulus. If the monkeys learned to associate each choice stimulus with its paired predictor, then response times to the choice stimuli should be faster in valid trials compared with the probe trials. In this sense, the design is similar to the cued target detection task used to study spatial attention by Posner et al. (1980), except that in this case the cues and targets were specific patterns rather than specific spatial locations. We refer to this task as the "passive paired-associate task" because in contrast to the standard pairedassociate task, it does not require active memory for the stimulus pairs and the subjects may not even be aware of the pairings.

Task description. Monkeys initiated each trial by grabbing a bar in the front of a standard monkey chair for $200 \mathrm{msec}$, after which a fixation spot $\left(0.1^{\circ}\right.$ in diameter) appeared in the center of the monitor (task time line is illustrated in Fig. 1A). Approximately $300 \mathrm{msec}$ after the monkeys fixated the spot, one of eight predictor stimuli appeared on the monitor for $500 \mathrm{msec}$, centered on the fixation spot. After a random delay period of 950-1050 msec, one of eight choice stimuli appeared at the fixation spot (a small subset of neurons was recorded with random delays of $500-1000 \mathrm{msec}$ ). Half of the choice stimuli were GO stimuli, and half were NO-GO. In the GO trials, the monkey was rewarded with a drop of orange juice if it released the bar within $100-1000 \mathrm{msec}$ after choice stimulus onset. To encourage rapid responses, two juice rewards were given if the monkey released the bar within $300 \mathrm{msec}$ of stimulus onset. The choice stimuli remained on for $500 \mathrm{msec}$ or until the monkey released the bar, whichever came first. Response latencies were defined as the time between the onset of the choice stimulus and the release of the bar. In NO-GO trials, the choice stimulus remained on for $500 \mathrm{msec}$, and the monkeys were rewarded for not releasing the bar between 100 and $1000 \mathrm{msec}$ after stimulus onset. Monkeys learned via trial and error which choice stimuli were GO and which were NO-GO. Trials were aborted if the monkey broke fixation at any time during the trial before the bar release or if it released the bar before or within the first $100 \mathrm{msec}$ of choice stimulus presentation (Fig. $1 B$ ). These trials are referred to as valid because a given predictor is paired with its associated choice stimulus in $80-90 \%$ of the trials.

Probe trials. The remaining two types of trials were probe trials, "invalid" and "neutral." Probe trials were added to the task after the monkeys were performing at or above $85 \%$ correct for the valid trials. The purpose of the probe trials was to enable us to measure the difference between the behavioral response latencies in trials with expected stimuli (valid trials) and behavioral latencies in trials with unexpected stimuli.

In the invalid probe trials, both the behavioral response and the choice stimulus were different from that of valid trials. In such trials, a NO-GO choice was preceded by a predictor stimulus that normally predicted a $\mathrm{GO}$ choice stimulus, or a GO choice stimulus was preceded by a predictor stimulus that normally predicted a NO-GO choice stimulus (Fig. 1C). When it saw the predictor stimulus in an invalid trial, the monkey might prepare a motor response and later suppress it, or the monkey might fail to prepare a motor response and then later have to prepare and execute a bar release. Slower response times in the invalid GO trials compared with that in valid GO trials provided a measure of association learning for specific predictor-choice pairings in addition to an indication of the time required to reprogram the behavioral response. Because association learning and motor preparedness are confounded in this type of probe trial, we also included neutral probe trials.

In neutral probe trials, a GO choice stimulus was preceded by a predictor that normally predicted a GO choice, and a NO-GO choice stimulus was preceded by a predictor that normally predicted a NO-GO choice, but in each case the specific predictor-choice pairing was different from that of valid trials (Fig. $1 D$ ). For example, consider the predictor-choice pairings of $\mathrm{A}-\mathrm{B}$ and $\mathrm{C}-\mathrm{D}$ on valid trials, with $\mathrm{B}$ and $\mathrm{D}$ both GO choice stimuli. In neutral trials, the stimuli would be repaired as A-D and $\mathrm{C}-\mathrm{B}$. Slower response times in the neutral GO trials, compared with that in valid GO trials, provided a measure of association learning for specific predictor-choice pairings without the additional factor of response reprogramming. All choice and predictor stimuli used in the session appeared in all three types of trials, i.e., valid, neutral, and invalid.

\section{Surgery}

Before surgery, the monkeys were placed in a plastic stereotaxic frame and scanned with magnetic resonance imaging (MRI). Appropriate stereotaxic coordinates for the recording chamber were calculated from the MRI scans. During surgery, under isoflurane anesthesia, a recording chamber was implanted on the dorsal surface of the skull over the perirhinal cortex. In addition, a post for restraining the head and a magnetic search coil for monitoring eye movements were implanted (Robinson, 1963). Analgesics and antibiotics were administered during the recovery period.

\section{Localization of electrode tracks}

Electrode placement into the perirhinal cortex was guided by constructing individual brain atlases from the MRI scans (Alvarez-Royo et al., 1991). Electrode locations were verified with $\mathrm{x}$ rays [similar to but simplified from the method of Nahm et al. (1994)]. Comparisons between $x$ rays and MRI scans were made by identifying the stereotaxic plane corresponding to the line between the auditory canal and the orbital ridge. The electrodes themselves were too small to be seen on the $\mathrm{x}$ rays, but the guide tubes were clearly visible, and the distance from the tip of the electrode to the tip of the guide tube was known. The depth and the anterior-posterior coordinates of the probes were verified from the sagittal-view x rays, and the medial-lateral coordinates were measured relative to the midline from the coronal-view $x$ rays. Histological examination of the brain tissue was not possible because both monkeys are still alive.

\section{Electrophysiological recording techniques}

Electrodes. Individual neurons were recorded with either tetrodes or single sharpened tungsten electrodes (ROBOZ Microprobe, Rockville, $\mathrm{MD}$ ). Tetrodes were constructed by twisting together four strands of wire and attaching them to a multipin connector with conductive epoxy or silver print. Two types of wire were used for the tetrodes: $20 \mu \mathrm{m}$ lacquer-coated tungsten wire (California Fine Wire, Grover City, CA) or $12.5 \mu \mathrm{m}$ Teflon-coated nichrome wire (H. P. Reid Company, Neptune, NJ). The Teflon-insulated tetrode wires were held together by warming the insulation briefly until it reached a tacky state. The lacquer-coated wire was held in place with a small amount of superglue. Nichrome wire was gold plated before use to reduce the electrode impedance. Electrode impedance ranged from 100 to $500 \mathrm{k} \Omega$ and from $500 \mathrm{k} \Omega$ to $1 \mathrm{M} \Omega$ for the tungsten and nichrome tetrodes, respectively. Impedance averaged $1 \mathrm{M} \Omega$ for the standard tungsten electrodes.

Microdrives. Tetrodes were advanced using minimicrodrives [similar to those described by Nichols et al. (1998)] attached to a cylindrical grid that fit snugly into a standard recording chamber (Crist et al., 1988). Briefly, an arm was attached to a threaded rod that was inserted into the grid. One rotation of the screw moved the arm $300 \mu \mathrm{m}$. Tetrodes were lowered into the perirhinal cortex through the grid via a telescoping guide tube system. The grids and minimicrodrives could remain in place for as long as 1 month; however, the electrodes could be moved on a daily basis so that different neurons were recorded each day. A standard manually driven hydraulic microdrive was used to manipulate the sharpened tungsten electrodes (for details, see Miller et al., 1993).

\section{Data collection and analysis}

A multipin head stage connected the tetrodes to a multichannel amplifier. Signals were amplified from $\sim 10,000$ to 23,000 times and bandpass filtered from $300 \mathrm{~Hz}$ to $8 \mathrm{kHz}$. Waveforms from all four channels of a tetrode were collected if one of the channels crossed a threshold. Waveforms were digitized at $25 \mathrm{kHz}$ and stored on computer disk for off-line spike sorting (Datawave Technologies, Longmont, $\mathrm{CO}$ ). Individual neurons were identified on the basis of their relative amplitudes or widths from the different tetrode channels (McNaughton et al., 1983; Gray et al., 1995).

Data were collected if at least one of the neurons appeared to be responsive to some aspect of the task. Neuronal responses to stimuli were measured as the average firing rate $(\mathrm{Hz})$ during a $175 \mathrm{msec}$ epoch starting $75 \mathrm{msec}$ and ending $250 \mathrm{msec}$ after stimulus onset (before the monkey's behavioral response). The very few trials in which the monkey responded in $<250 \mathrm{msec}$ were eliminated from further analysis. Delay activity was measured as the mean firing rate starting $200 \mathrm{msec}$ and ending $800 \mathrm{msec}$ after the predictor stimulus was turned off. These fixed epoch windows were used for all calculations.

Stimulus selectivity was assessed using ANOVAs (evaluated at $p<$ $0.05)$ calculated on the trial-by-trial data for each neuron to the eight predictor and choice stimuli separately, using the time windows described above. Delay period selectivity was determined in the same way for the delay periods after each of the eight predictor stimuli. A measure of stimulus selectivity $\left(r^{2}\right)$ was calculated from the ANOVA table for each neuron by simply dividing the sum of squared deviations for the 
treatment by the total sum of squared deviations from the grand mean (Keppel and Zedeck, 1989). The $R^{2}$ statistic provides an estimate of how much of the variance in firing rate can be accounted for by specific stimuli and, unlike $F$ ratios and $p$ values, is not influenced by sample size. The normalized magnitude of the neuronal response was measured by computing a $z$ score for each stimulus relative to the distribution of baseline firing rates.

Response similarity to paired stimuli was measured as the correlation between the mean responses to the predictor and choice stimuli for each pair. A large $r$ value indicates that the responses to predictor and choice stimuli were similar, such that if a neuron responded well to one stimulus in a pair then it also responded well to the other of the pair. Correlations were also computed between the firing rate during the delay and the response to the preceding (predictor) stimulus and the following (choice) stimulus. All $r$ values were converted to Fisher $z$ scores before statistical analysis.

\section{RESULTS}

\section{Behavioral performance}

The monkeys typically learned eight new choice stimuli to a criterion of $85 \%$ correct in $<250$ trials or $\sim 30$ presentations per stimulus. Learning curves are shown in Figure $2 A$. The percent correct for each trial type was calculated after the performance reached asymptote and probe trials were added. The percent correct (Fig. $2 B$ ) for the data sets recorded with familiar stimuli $(97.9 \% ; n=87)$ was slightly, but significantly, better than that for the novel $(90.4 \% ; n=41)$ stimuli $\left[F_{(1,126)}=29.7 ; p<0.0001\right]$. There was a small, but significant, difference in the percent correct for the invalid trials compared with either the neutral or valid trials [mean percent correct values for valid, neutral, and invalid trial types were 95.5, 88.6, and 97.1\%, respectively; $\left.F_{(2,252)}=44.8 ; p<0.0001\right]$. That is, mistakes were more likely when the rewarded behavioral response was unexpected. Although both predictor type and experience influenced the mean response latencies, there was no interaction between these two factors $\left[F_{(2,252)}=1.3 ; p=0.268\right]$. Overall, the monkeys learned the stimuli quickly and performed at a very high level.

\section{Response latencies}

Behavioral evidence of association learning was determined by the differences in response latencies to expected (valid trials) and unexpected (invalid and neutral trials) stimuli. The response latencies to the familiar stimuli remained relatively stable throughout the session. However, the response latencies to the novel stimuli were, on average, $100 \mathrm{msec}$ slower than the latencies to the familiar stimuli at the beginning of the data set and declined over the course of the recording session, approaching (but not reaching) the mean latency of the familiar stimuli after 500 trials (Fig. 2C).

The relationship between the effect of experience (novel and familiar) and predictor type (valid, invalid, and neutral) on response time latencies was determined by computing a two-way ANOVA, with predictor type as a within-session factor and experience as a between-session factor. There was no significant interaction between the factors $\left[F_{(2,252)}=2.5 ; p=0.082\right]$. There were, however, significant main effects for both predictor types $\left[F_{(2,252)}=117.2 ; p<0.0001\right]$ and experience level $\left[F_{(1,126)}=20.1\right.$; $p<0.0001]$. The mean response latencies were slightly faster to familiar stimuli than were the responses to the novel stimuli even after the percent correct for the novel stimuli reached the level of the familiar stimuli. The mean response time latencies for the valid trials were 398.3 and $482.6 \mathrm{msec}$ for the familiar and novel stimuli, respectively, after probe trials were added. For both the novel and familiar stimuli, the monkeys' response latencies were shorter for the valid compared with the neutral trials, suggesting
A

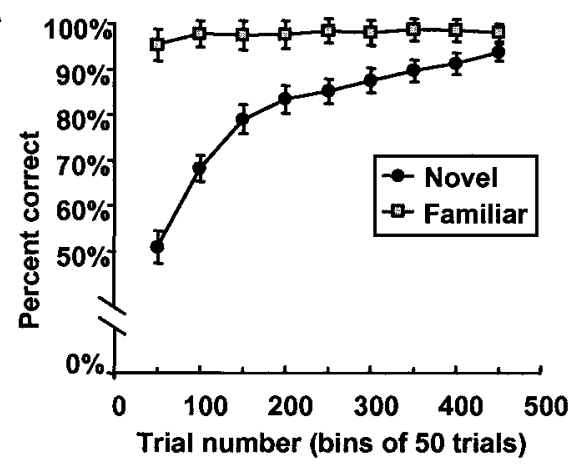

B

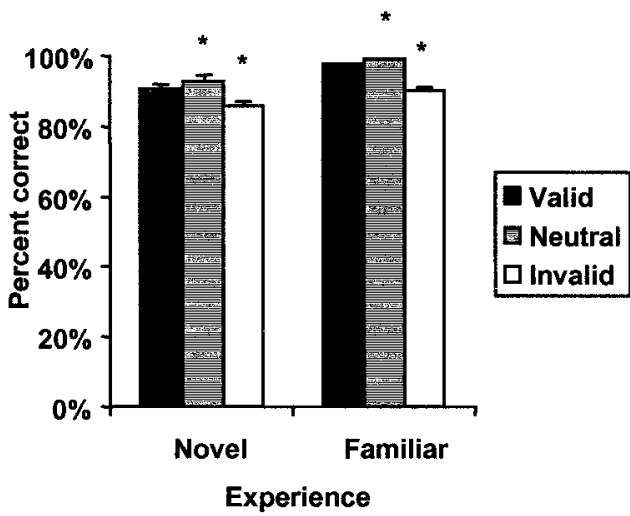

C

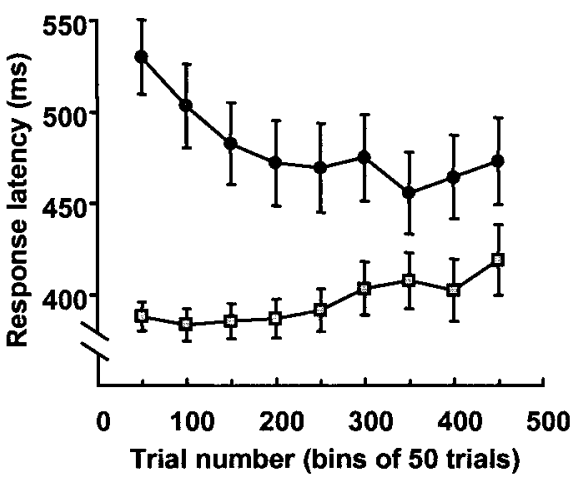

D

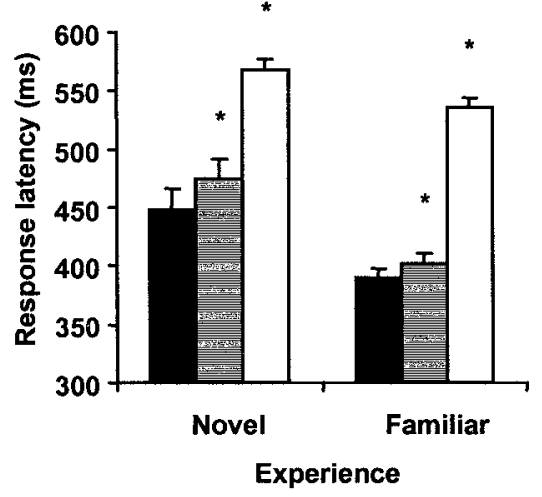

Figure 2. Behavioral performance. A, Percent correct in 50 trial bins for the first 500 trials for each recording session. Data are shown separately for novel and familiar stimuli. $B$, Average percent correct in valid, invalid, and neutral trials. Asterisks indicate a significant difference from the performance in the valid trials $(t$ test, $p<0.05)$. $C$, Reaction times for 50 trial bins, during the first 500 trials of the recording sessions. Symbols are described in $A$. $D$, Mean reaction times in valid, invalid, and neutral trials. Asterisks indicate a significance difference from the reaction times of valid trials $(t$ test, $p<0.05)$. Vertical bars are described in $B$. Error bars in these, and subsequent, graphs indicate the SEM. 


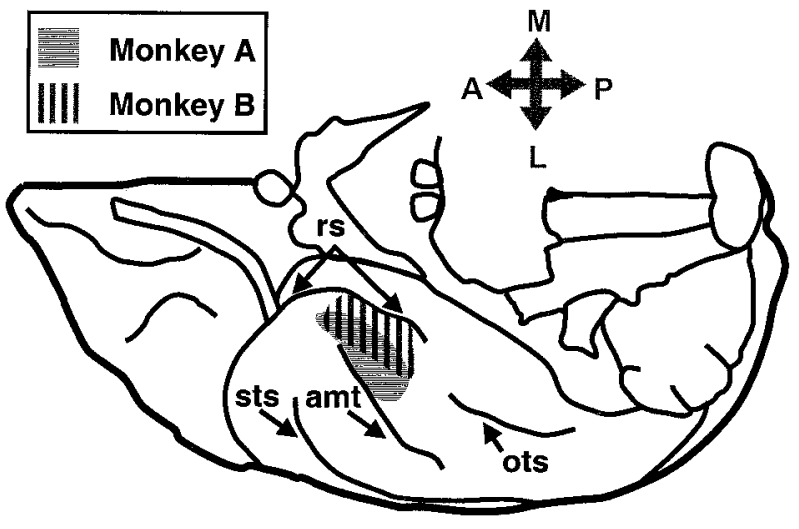

Figure 3. Location of recording sites on the ventral view of the brain. The recording regions for both monkeys are shown in the same hemisphere in this illustration; however, monkey $A$ was recorded from the right hemisphere, and monkey $B$ was recorded from the left hemisphere. The recording sites for both monkeys are shown in different shading patterns. $A$, Anterior; $L$, lateral; $M$, medial; ots, occipital temporal sulcus; $P$, posterior; sts, superior temporal sulcus.

that the monkeys did learn to associate the predictor stimuli with the paired choice stimuli as well as with the ultimate behavioral response after the choice.

The mean response times were 389.3, 402.4, and $536.6 \mathrm{msec}$ for valid, neutral, and invalid trials, respectively, for the familiar stimuli (Fig. 2D). Subsequent contrast computations indicated that the response time difference between valid and invalid trials was significant at both experience levels. Most importantly, the response time difference between valid and neutral trials was significant for both the novel (mean latency difference $=25.5 \pm$ $11.0 \mathrm{msec} ; p=0.026$ ) and familiar (mean latency difference $=$ $13.1 \pm 4.0 \mathrm{msec} ; p=0.002$ ) stimulus pairs. These results indicate that the monkeys learned the stimulus pair associations for both novel and familiar stimuli.

It was not possible to pinpoint the exact time the monkeys learned the association between the two stimuli. The probe trials were added only after the monkeys were performing at $>85 \%$ in the valid trials. There was a much smaller number of probe trials in each data set compared with the valid trials; thus, it was not possible to track the learning on a trial-by-trial basis. The latency differences between the valid and neutral trials were significantly different in $\sim 25 \%$ of the recording sessions for both novel and familiar recording sessions.

\section{Anatomical location of electrode penetrations}

The majority of penetrations were made within the boundaries of the perirhinal cortex as defined by Suzuki and Amaral (1994), except for two penetrations made in TE, near the anterior middle temporal sulcus, in monkey $A$ (Fig. 3). The estimated recording areas for both monkeys are shown in Figure 3.

\section{Basic response properties of neurons}

Of the 386 neurons recorded, 127 were recorded while monkeys learned new stimuli (novel). The remaining 259 neurons were recorded during presentation of familiar stimuli. Of the cells studied with familiar and novel stimuli, 47 and 52\%, respectively, showed significant stimulus selectivity responses to both predictor and choice stimulus sets, according to one-way ANOVAs.

The basic stimulus-selective properties of the neurons did not differ for cells tested with novel and familiar stimuli (Fig. 4). The mean firing rate was higher for cells tested with novel compared
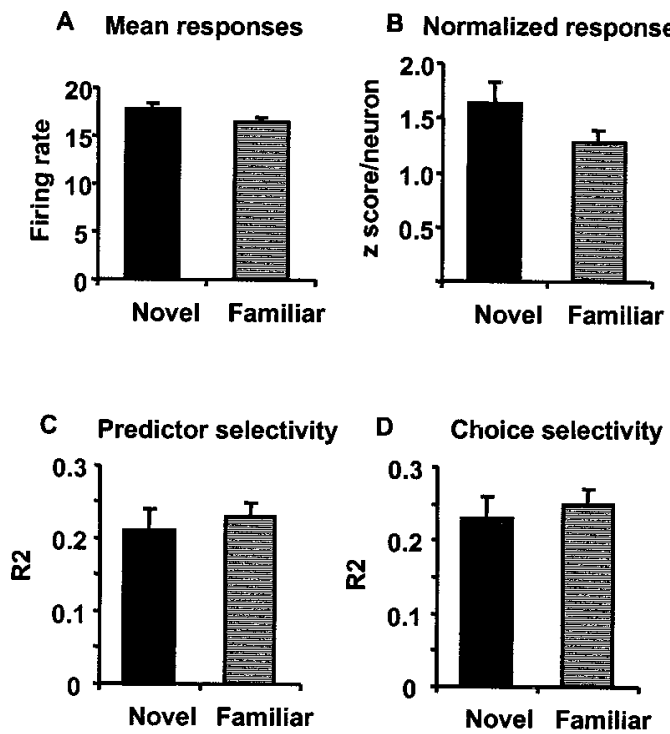

Figure 4. Basic neuronal response properties for novel and familiar stimuli. $A$, Neuronal responses, as measured by the mean firing rates, were not different between novel and familiar stimuli. $B$, The normalized responses, as measured by $z$ scores, were slightly but not significantly smaller for the familiar compared with the novel stimuli. $C, D$, But there was no difference in the amount of the response variance $\left(r^{2}\right)$ accounted for by individual predictor $(C)$ or choice $(D)$ stimuli between the novel and familiar stimuli.

with familiar stimuli, and the number of stimuli that elicited a significant response ( $z$ scores above the baseline firing rate) was higher for cells studied with novel stimuli, but both of these differences just failed to reach conventional measures of significance $[t(2990)=1.61 ; p=0.054 ; t(372)=1.64 ; p=0.051]$. Likewise, there was no significant difference between novel and familiar stimuli in the amount of the variance in firing rate, on a trial-to-trial basis, accounted for by the specific stimuli $\left(r^{2}, t<1\right)$. Although other studies have found significant decreases in inferior temporal responses as novel stimuli have become familiar within a single recording session (e.g., Fahy et al., 1993; Li et al., 1993), these decreases were apparently not large enough to cause substantial differences in firing rates between the cells studied in the first versus subsequent recording sessions in the present study.

\section{Experience-dependent changes in responses to stimuli} To test whether training on the paired associates altered the selectivity of the cells, we examined the pattern of stimulus selectivity for the predictor and choice stimuli. The analysis was directed at the 66 and 121 cells that gave stimulus-selective responses to the novel and familiar stimuli, respectively. An example of one cell's responses to the predictors and choices is shown in Figure 5. As the figure demonstrates, the cell's responses to the eight predictor stimuli varied from a good response (predictor stimulus 5) to virtually no response (predictor stimulus 4). Importantly, the responses to the associated choice stimuli followed the same order; i.e., the responses to the predictor and choice stimuli were highly correlated. The Pearson correlation coefficient for the responses to predictors and associated choices was 0.85 for this cell (see Fig. $5 B$ ).

The distribution of $r$ values for the entire population of selective cells is shown separately for novel and familiar stimuli in Figure 6. Although the responses of any given cell to the predictor and choice stimuli might be correlated by chance, the stimuli for 

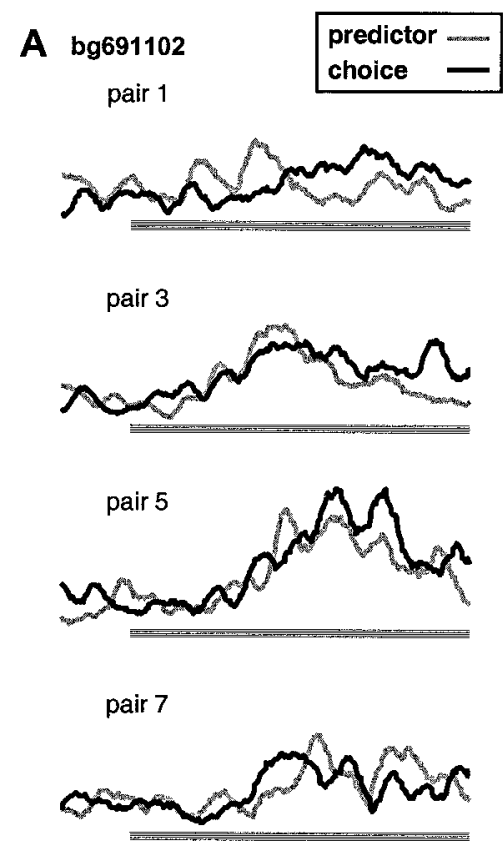

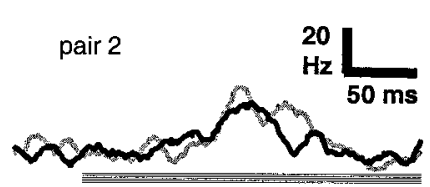

pair 4
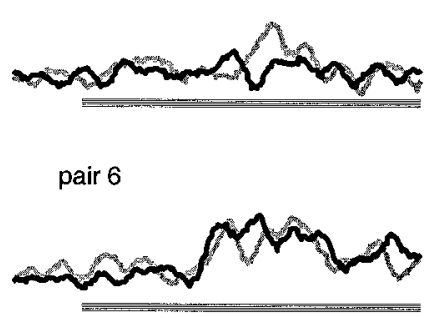

pair 8

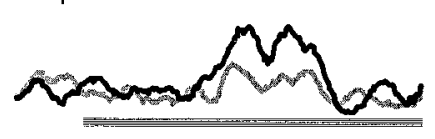

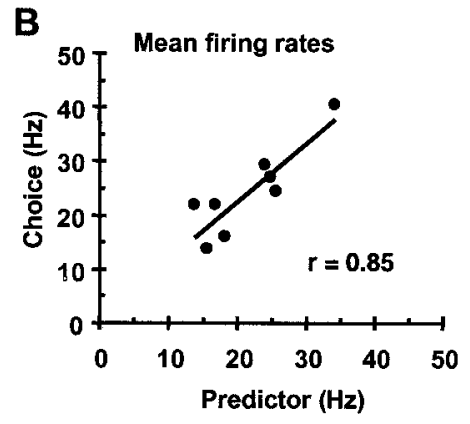

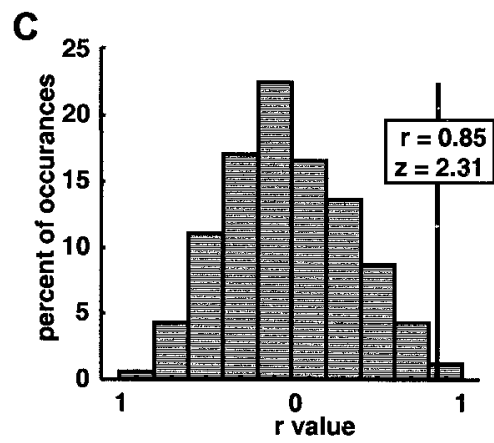

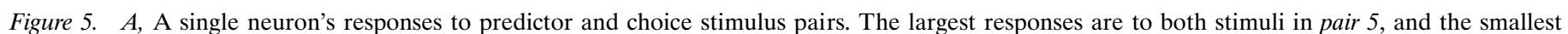

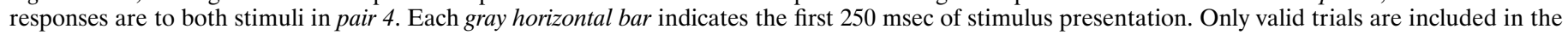

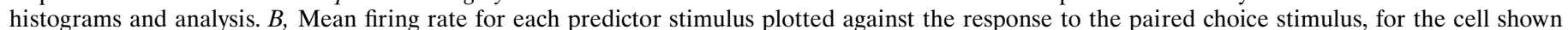

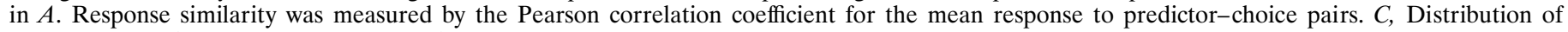
correlation coefficients for randomly shuffled pairs compared with the actual stimulus pairs for the same cell.

each cell were chosen randomly; thus, the mean of the distributions of $r$ value should be 0 if association learning did not influence the relative responses to the predictors and choices. As can be seen in the figure, the distribution of $r$ values for the novel stimuli was very close to 0 (mean $r=-0.002$ ), which was not different from chance $[t(65)=0.32 ; p=0.974]$. However, the distribution of $r$ values for the familiar stimuli was positive (mean $r=0.145)$ and significantly different from $0[t(120)=3.65 ; p<$ 0.0001]. Furthermore, the means of the distributions of $r$ values for the novel and familiar stimuli were also significantly different from one another $[t(185)=2.28 ; p=0.024]$. Thus, the association learning appeared to cause the responses to paired predictor and choice stimuli to become more similar to one another for the familiar, but not the novel, stimuli (all statistical analyses were performed on Fisher $z$ scores, not on $r$ values). Furthermore, the average amount of the variance in mean responses to choice stimuli accounted for by the responses to the predictor stimuli was greater for familiar stimuli $\left(R^{2}=0.184 \pm 0.019\right)$ than for novel stimuli $\left(R^{2}=0.131 \pm 0.022\right)$.

To check further the distributions of $r$ values against chance, we randomly shuffled the pairings between predictor and choice stimuli for each cell 1000 times and computed a correlation coefficient each time. This gave us a random distribution of $r$ values for each cell, which enabled us to measure the difference between the actual $r$ value and the mean of the shuffled values in $z$-score units. The $z$ score of the $r$ value for the cell shown in Figure 5, for example, was 2.31, which was significantly different from 0 ( $p<0.010$; see Fig. $5 C)$. There were a total of four neurons with significant $z$ scores (evaluated at $p<0.05$ ) with novel stimuli, which was not different from chance according to a binomial test $\left(X^{2}=0.09 ; p>0.05\right)$. However, there were 17 neurons with significant $z$ scores with familiar stimuli, which was significantly greater than chance (binomial test, $X^{2}=5.0 ; p<$
0.05). Again, these results indicate that association learning caused the responses to predictor and choice stimuli to become more similar with prolonged association learning for at least some cells.

We also added all of the shuffled $r$ distributions from all cells into population distributions of shuffled values, which are shown in Figure 6. The means of the shuffled distributions for both novel and familiar stimuli were both 0 , as expected. Comparing the shapes of the distributions for the actual and shuffled $r$ values across the population, there appears to be an excess of cells with high actual $r$ values, but this is only true for cells tested with familiar stimuli.

\section{Classifying neurons by behavioral evidence of association learning}

Because the behavioral task did not actually require that the animals learn to associate the predictor and choice stimuli, it was possible that the animals learned to associate the pairs in some recording sessions but not in others. If so, then it was also possible that the effects of association learning on responses would be larger in those recording sessions in which the reaction time data demonstrated learning than in those sessions in which there was no significant difference between reaction times to valid and neutral probes. To test this, we grouped cells according to whether or not the monkey showed significantly increased response latencies during neutral probe trials compared with valid trials. It was possible to classify 38 stimulus-selective neurons recorded during presentation of novel stimuli and 66 neurons during presentation of familiar stimuli. The relationship between experience and behavioral evidence of association learning was tested with a two-way ANOVA. There was a main effect of experience [novel vs familiar; $F_{(1,170)}=4.3 ; p=0.039$ ], but there was no significant effect of behavioral evidence of association 

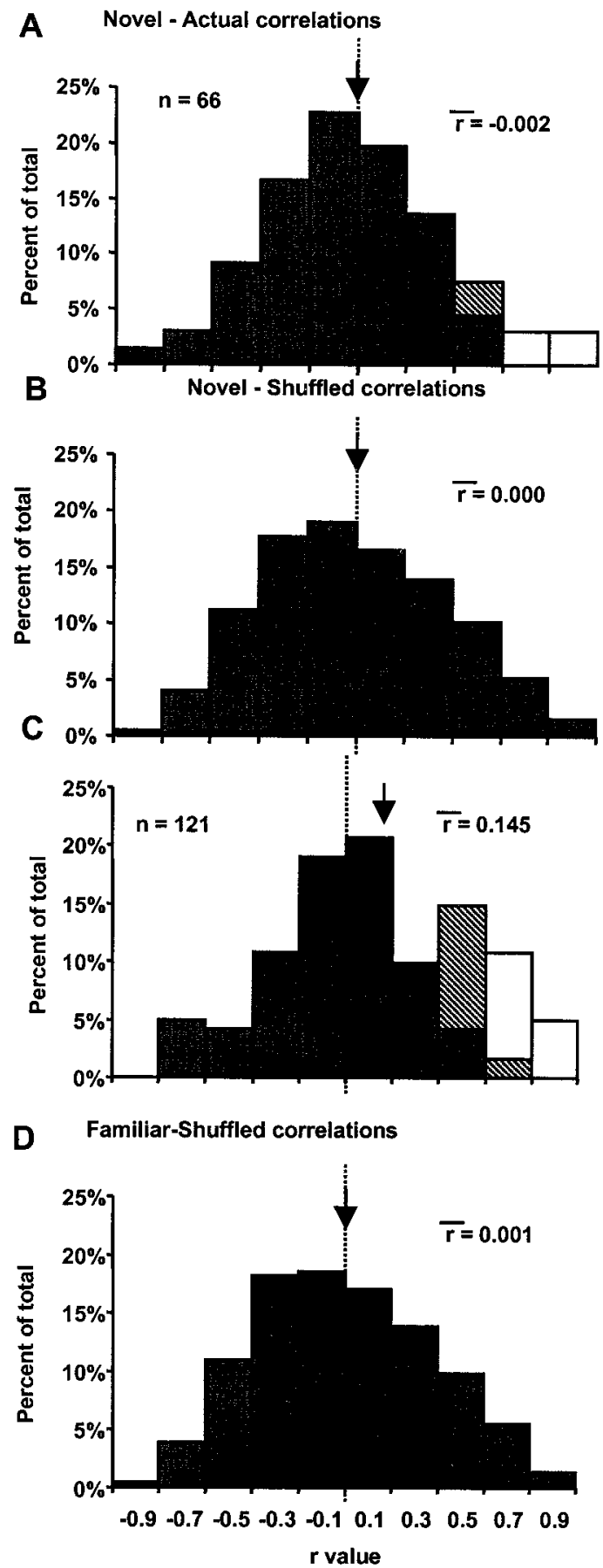

Figure 6. Distrubution of actual and shuffled stimulus pair correlation coefficients. $A$, The distribution of correlation coefficients was not different from chance for the novel stimuli; i.e., there were just as many neurons with negative correlations as there were with positive correlations. The white vertical bars indicate neurons with $z$-score significance levels at $p<$ 0.05 , and the diagonal-striped vertical bars indicate $z$ scores with $p<0.10$. $B$, The mean correlation for the novel paired stimuli was not different from the mean for the shuffled pairs. $C$, In contrast, the responses of the neurons to the pairs of familiar stimuli were more likely to be positively correlated; i.e., the paired stimuli had similar responses (even though they were visually quite distinct). $D$, The mean distribution for the familiar shuffled pairs is shown. There was no difference in the distribution of novel and familiar correlations for the distribution of shuffled stimulus correlations. The vertical dotted line is above 0 in all four histograms. Labels on the $x$-axis indicate the center of the bin. Mean $r$ values (indicated by arrows) and the number of neurons for each group are indicated on the graphs.

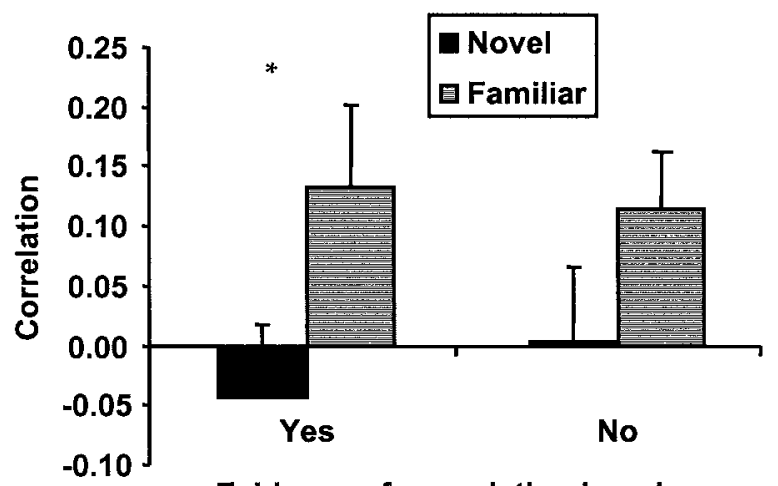

Evidence of association learning

Figure 7. Correlation between responses to paired predictor and choice stimuli, shown separately for recording sessions in which the animal did (Yes) and did not $(\mathrm{No})$ show behavioral evidence of association learning. Behavioral evidence consisted of significant reaction time differences for valid versus neutral stimulus pairs.

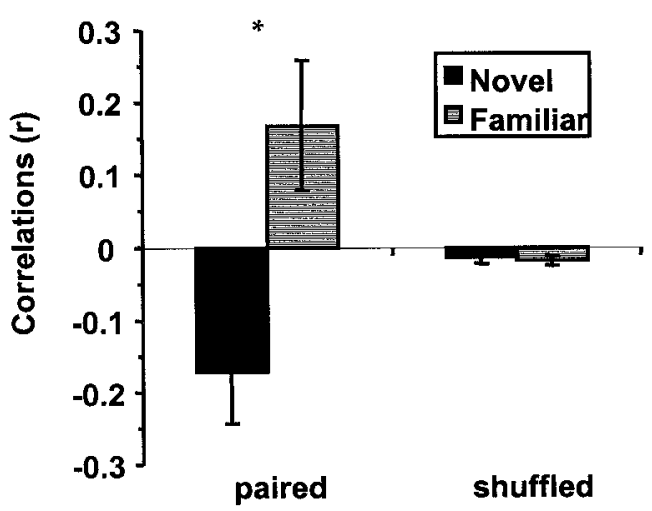

Figure 8. Correlation between responses to paired predictor and choice stimuli, for seven cells studied with both novel and familiar stimuli. The mean correlation differed significantly for the novel and familiar stimuli $(t$ test, $p<0.05)$. The mean correlation between predictor and choice stimuli was near 0 for randomly paired stimuli.

learning ( $p=0.845)$ and no significant interaction between the two factors $(p=0.646)$. Experience, i.e., having learned the stimuli on a previous day, was the only significant factor in the increased stimulus pair correlations (Fig. 7).

\section{Neurons recorded with both novel and familiar stimuli}

One possible, but unlikely, explanation for the difference in response correlations between novel and familiar stimulus pairs is that there might have been systematic differences between the neurons studied with familiar stimuli compared with neurons studied with novel stimuli. To address this issue, seven stimulusselective neurons were tested with both novel and familiar stimuli. These neurons had a positive mean correlation $(r=0.169)$ for responses to the familiar stimulus pairs and a negative mean correlation $(r=-0.172)$ for responses to the novel pairs (Fig. 8). The difference between the two experience conditions was significant [mean difference $=0.341 ; t(12)=2.97 ; p=0.012$ ]. Thus, even when the same cells were tested with both types of stimuli, there was evidence of association learning in the responses to the familiar stimuli but not the novel ones.

\section{Delay activity}

A subpopulation of the stimulus-selective neurons also responded differentially during the delay after the predictor stimulus and 

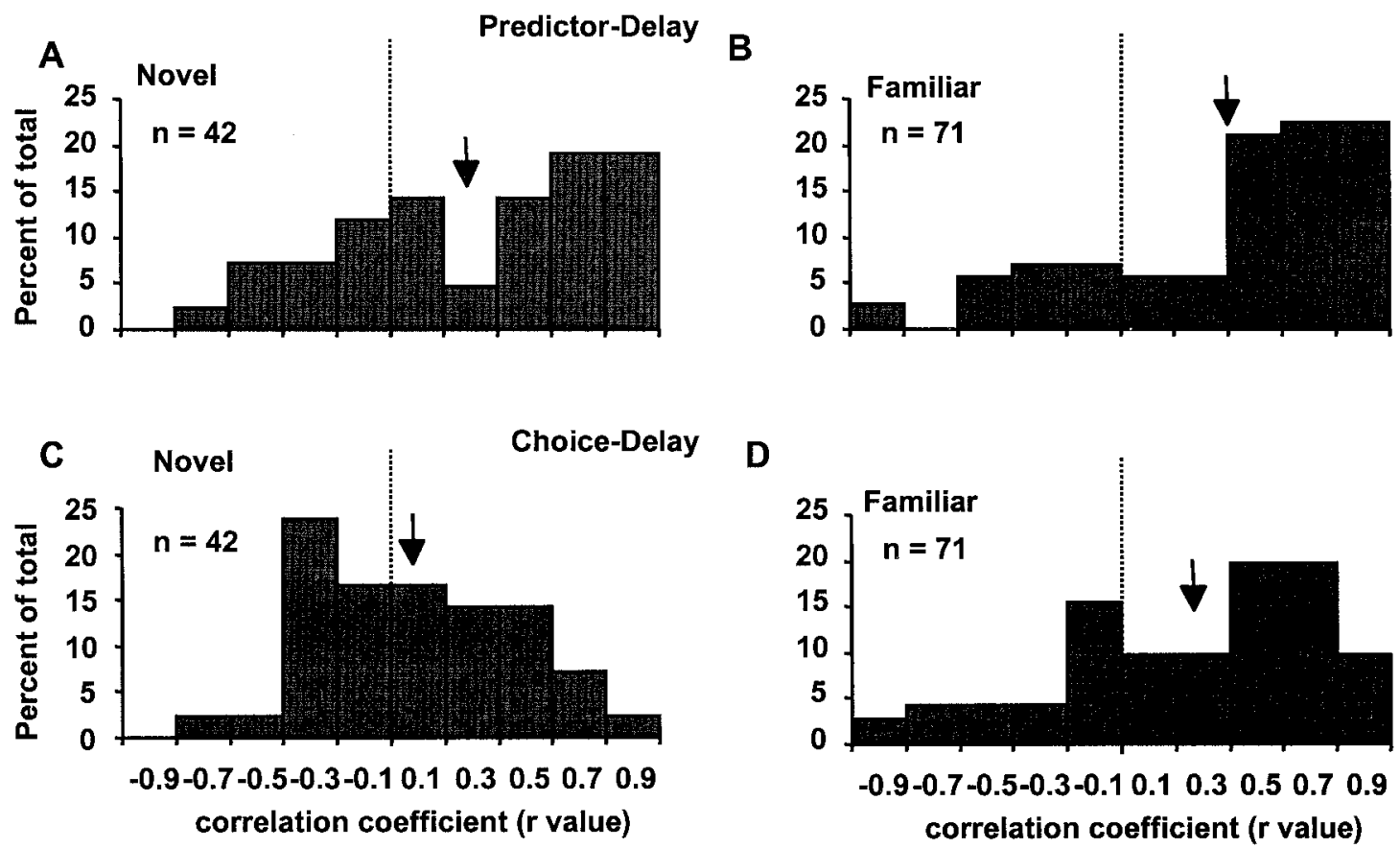

Figure 9. A, B, Correlations between the magnitude of the delay activity and the magnitude of the response to the predictor stimulus, shown separately for cells studied with novel stimuli and cells studied with familiar stimuli. The mean correlations did not differ significantly for novel versus familiar stimuli $(p=0.34)$. $C, D$, Correlations between the magnitude of the delay activity and the magnitude of the response to the choice stimulus, shown separately for cells studied with novel stimuli and cells studied with familiar stimuli. The mean correlation for familiar stimuli was significantly greater than that for novel stimuli $(p=0.03)$.

before the choice stimulus. This subpopulation was identified via ANOVAs calculated on the firing rates during the delay period. All neurons included in this analysis were selective during each of the three time periods of a trial (predictor, delay, and choice). Of the 66 stimulus-selective cells studied with novel stimuli, 42 (63.6\%) showed significant stimulus selectivity during the delay period, as did $71(58.7 \%)$ of the 121 stimulus-selective cells studied with familiar stimuli.

It has been shown previously in DMS tasks that the magnitude of delay activity after different predictor stimuli is correlated with a cell's selectivity for the different predictors, i.e., that the delay activity is determined by a "retrospective" memory of the predictor stimulus (Fuster and Jervey, 1981, 1982; Miller et al., 1993). For example, such a cell would have the highest amount of delay activity after a predictor stimulus that elicited a good response and the least delay activity after a predictor stimulus that elicited a poor response. By contrast, it has been reported that in pairedassociate learning tasks, the magnitude of the delay activity between the predictor and the choice is determined by the cell's selectivity for the choice stimulus, i.e., that the delay activity is determined by a "prospective" memory for an expected stimulus (Sakai and Miyashita, 1991; Naya et al., 1996). To test for these two possibilities, for each neuron we calculated the Pearson correlations between the firing rate during the delay period and the magnitudes of the responses to both the predictor and choice stimuli.

\section{Retrospective delay activity}

The distributions of correlation values between the responses to the stimuli and the activity during the delay are shown in Figure 9 for the population of cells. Delay activity was correlated with the magnitude of response to the predictor stimulus for both the novel (mean $r=0.316$ ) and familiar (mean $r=0.404$ ) stimuli.
Both of these distributions were different from 0 [novel, $t(41)=$ 4.38; $p<0.0001$; familiar, $t(70)=7.12 ; p<0.0001]$, and they were not significantly different from each other $[t(111)=1 ; p=0.340]$.

An example of a cell with delay activity correlated with its response to the predictor stimuli is shown in Figure $10 A-C$. This particular cell did not have correlated responses to the associated choice stimuli. The delay activity fell to near baseline firing rates after the offset of all of the predictor stimuli, but then it climbed again only in trials that began with a preferred predictor stimulus.

\section{Prospective delay activity}

The correlation between the firing rate during the delay and the responses to the choice stimuli was near chance for the novel stimuli [mean $r=0.079 ; t(41)=1.34 ; p=0.188$ ]. For the familiar stimuli, however, the correlation between the responses to the choice stimuli and the firing rate during the delay was significantly greater than chance [mean $r=0.269 ; t(70)=4.75 ; p<0.0001$ ]. Furthermore, the familiar choice-delay correlations were significantly greater than the novel choice-delay correlations $[t(111)=$ 2.20; $p=0.030]$. An example of a cell with a positive correlation between the magnitude of delay activity and its responses to both the predictor and choice stimuli is shown in Figure $10 \mathrm{D}$.

In summary, for the novel stimuli, the magnitude and selectivity of the delay activity appeared to be determined by the stimulus the monkey had just seen. However, the delay activity for familiar stimuli was correlated with the responses to both predictor and choice stimuli. In other words, prolonged association training had no effect on retrospective delay activity but appeared to increase the likelihood of prospective delay activity.

\section{Neurons tested with both novel and familiar stimuli}

We also examined the delay activity of the seven neurons tested with both novel and familiar stimuli (Fig. 11). As above, there was 
Figure 10. Spike density histograms of neurons exhibiting stimulus-selective delay activity. $A$, Each $\mathrm{col}$ ored line represents the response of the same neuron to one of eight different pairs of associated stimuli. The response to the best predictor stimulus in the set of eight (red line) returns to baseline after the stimulus is turned off and then climbs during the delay period. $B$, The enlarged scale for the delay activity of the same cell shows that the magnitude of delay activity is correlated with the responses to the predictor stimuli. $C$, Mean responses are shown to the predictor stimuli and choice stimuli, both plotted against the magnitude of delay activity, for the same cell shown in $A$ and $B$. Mean delay activity was calculated during the interval from 200 to $1000 \mathrm{msec}$ after the predictor stimulus was turned off. $D$, An example from a different neuron, in which the magnitude of response to the predictor, the magnitude of activity during the delay, and the magnitude of response to the paired choice stimulus were all correlated with one another, is shown. The stimulus presentation times are indicated by gray horizontal lines.

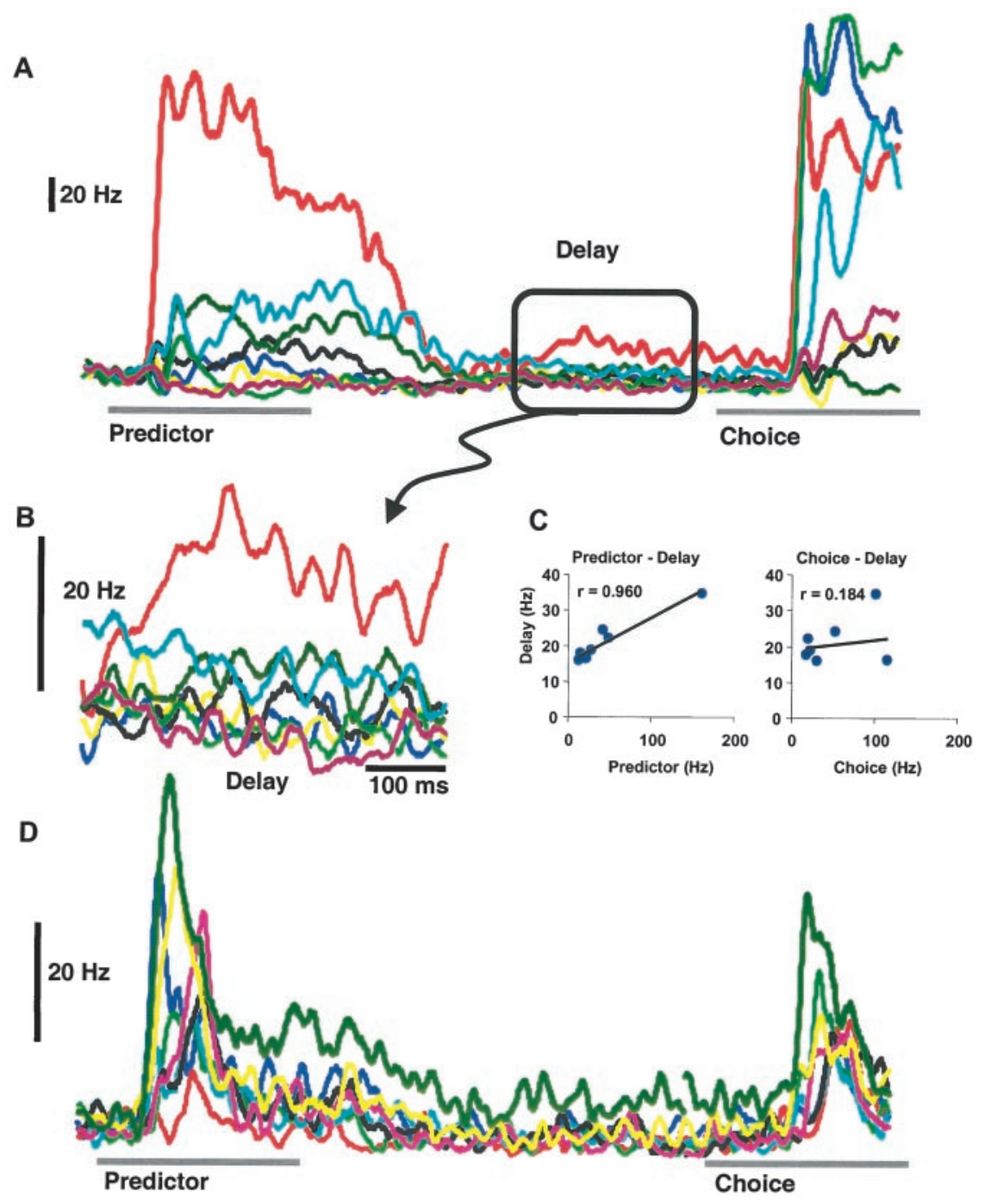

no effect of experience on the retrospective delay activity ( $r=$ 0.46 for novel compared with $r=0.30$ for familiar), but there was a large increase in prospective delay activity $(r=-0.31$ for novel compared with $r=0.39$ for familiar).

\section{Relationship between delay activity and} predictor-choice correlations

As described in the previous sections, some cells showed positive correlations between their responses to the predictors and choices, and some cells showed positive correlations between their delay activity and their responses to both the predictors and choices (Fig. 10D). This raised the possibility that the positive correlation between the delay activity and choice responses found for familiar stimuli was actually a side effect of the increased correlations between the predictor and choice responses found for familiar stimuli.

We used partial correlations to control statistically for the potential confound of the increased correlations between the responses to the stimulus pairs with experience (Keppel, 1973). This analysis provided a way to separate the correlation between predictor-choice responses from the correlation between choice responses and delay activity. The mean difference between the partial correlation and raw correlation coefficient was $0.065(t<$ $1 ; p=0.456)$ and $0.030(t<1 ; p=0.705)$ for the predictor-delay

\section{Delay Correlations}

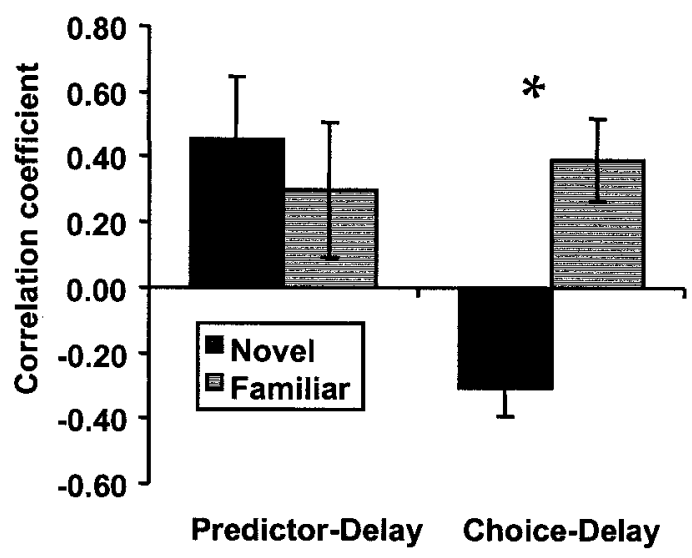

Figure 11. Correlation between the magnitude of response to the predictor and choice stimuli and the magnitude of the associated delay activity, for seven cells tested with both novel and familiar stimuli. The mean correlations differed significantly for novel versus familiar stimuli $(p<0.001)$. Delay-selective neurons tested with both novel and familiar stimuli showed the same shift in delay activity correlated with the choice stimuli $(n=7)$. Experience with stimuli changed the sign of the correlation $(p<0.001)$. 


\section{A Raw Correlations}

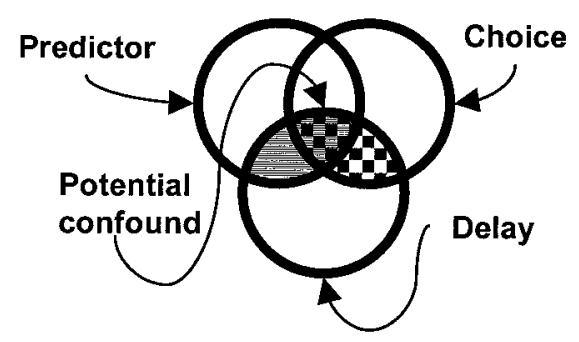

B Partial Correlations

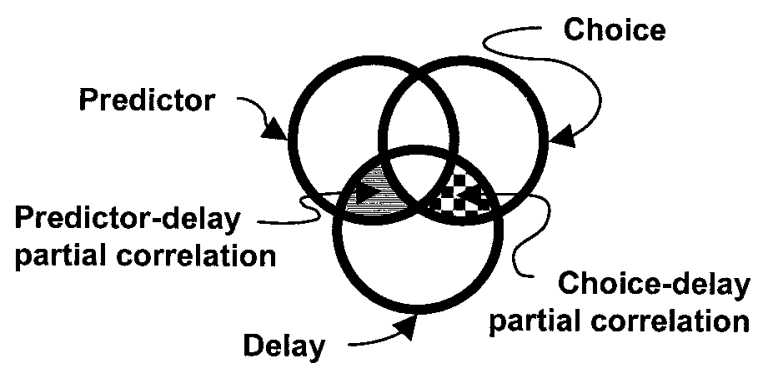

C
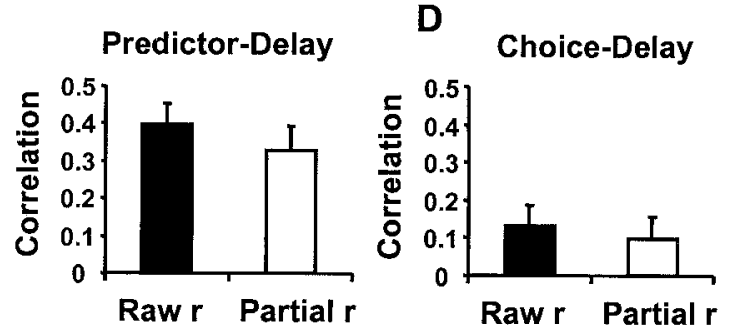

Figure 12. Partial correlations between the magnitude of response to the predictor and choice stimuli and the associated delay activity. $A, B$, The Venn diagrams conceptually describe the difference between the actual correlations and the partial correlations. $C$, $D$, Little of the predictordelay relationship can be accounted for by the predictor-choice correlations.

and choice-delay correlations, respectively. The predictorchoice relationship accounted for none of the retrospective delay activity and some, but not all, of the prospective delay activity correlations (Fig. 12). Thus, association learning appears to cause an increase in prospective delay activity that is not accounted for by the increased correlation between the responses to the predictor and choice stimuli.

\section{Classifying neurons by behavioral evidence of association learning}

Next we examined whether the relationship between the delay activity and stimulus-evoked responses varied according to whether or not the monkey showed behavioral evidence of association learning in a given recording session (Fig. 13). For the predictor stimuli, we conducted a two-way ANOVA on the correlations between the delay activity and the response to the predictor stimuli, with experience level (novel vs familiar) and evidence of association learning (reaction time difference between valid and neutral trials) as the two factors. The results indicated that experience level had no effect on the mean $r$ values (no main effect of experience, $p=0.348$ ). However, there was a significant main effect of association learning, with a mean $r$ of 0.429 in sessions with no evidence of association learning com-
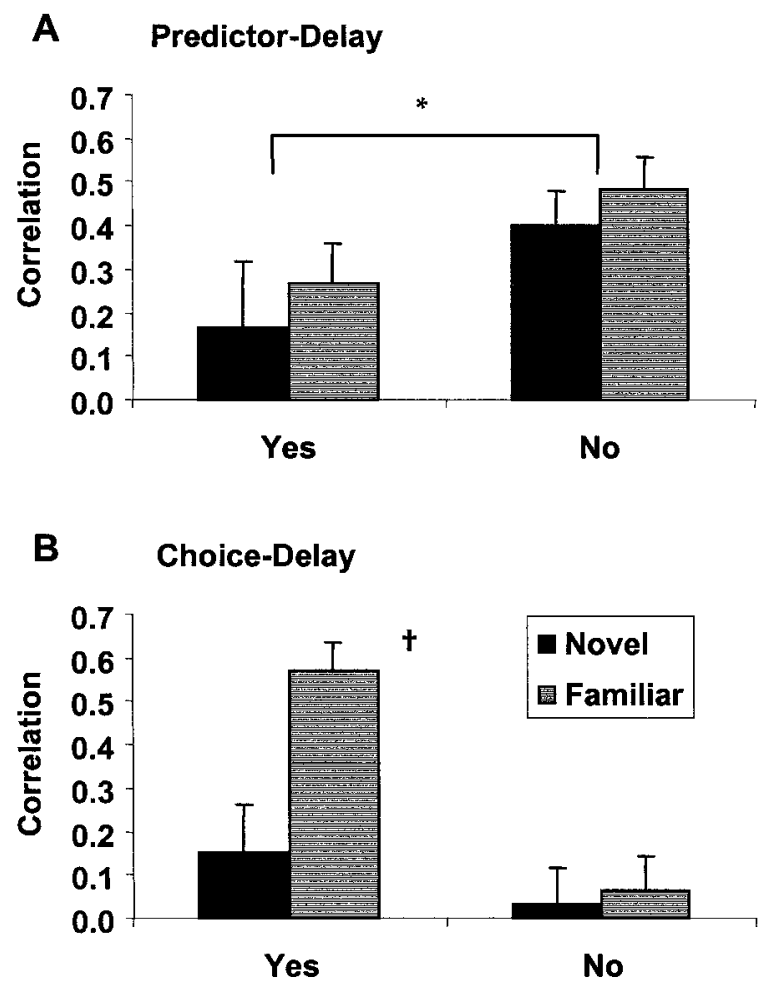

Evidence of association learning

Figure 13. Comparison of the effects of experience and behavioral evidence of association learning on delay activity. $A$, Correlation between delay activity and responses to the predictor stimuli. Delay activity was less tightly correlated with responses to the predictor stimuli for those data sets in which the monkeys showed evidence of association learning (main effect of learning, $p<0.05$ ). $B$, Correlations between delay activity and responses to choice stimuli. Delay activity was mostly tightly correlated with the responses to the choice stimuli in those sessions with behavioral evidence of association learning as well as in those sessions using familiar stimuli (main effect of both experience and learning).

pared with a mean $r$ of 0.232 in sessions with significant evidence of association learning $\left[F_{(1,100)}=5.2 ; p=0.024\right]$. There was no significant interaction between the effects of experience and association learning $(p=0.929)$. Thus, when the neurons were recorded during sessions in which there was evidence of association learning, the delay activity was actually less correlated with the response to the predictor stimulus (Fig. 13A). The reason for this superficially paradoxical result is suggested by the analysis below.

When we conducted the same analysis on the correlations between the delay activity and the responses to the choice stimuli, we found a complementary pattern of results (Fig. 13B). According to the two-way ANOVA, there were significant main effects of experience and association learning (experience $\left[F_{(1,100)}=6.6\right.$; $p=0.012]$; association learning $\left.\left[F_{(1,100)}=12.7 ; p=0.001\right]\right)$, and there was a significant interaction between the two factors $\left[F_{(1,100)}=4.8 ; p=0.032\right]$. In other words, in sessions with evidence of association learning, the correlation between delay activity and the response to the predictor stimuli fell at the same time that the correlation between the delay activity and the response to the choice stimuli rose. The highest correlations between the delay activity and responses to the choice stimuli were found for those neurons recorded with familiar stimuli and 
with behavioral evidence of association learning in the recording session (mean $r=0.572$ ).

In summary, on the first day of training, the responses to paired stimuli were uncorrelated, and the magnitude of delay activity was correlated almost solely with the response to the predictor stimulus, i.e., the stimulus that immediately precedes the delay. On subsequent days, the responses to paired stimuli became correlated, and the delay activity became correlated with the responses to both by the predictor and choice stimuli, i.e., the stimulus the animal has just seen and the stimulus to follow. In sessions with behavioral evidence of association learning, the delay activity tended to switch from being correlated with the predictor stimuli to being correlated with the choice stimuli. We take these shifts in the correlations with delay activity to indicate that as the monkey learns the relationship between the stimuli and uses the knowledge of the relationship to respond more quickly on valid trials, the delay activity shifts from reflecting the stimulus that was just seen to predicting the stimulus to come.

\section{DISCUSSION}

By using an associative-learning task that monkeys could learn quickly, we were able to measure separately neural correlates of associative memory for recently learned (i.e., novel) associates and well learned (i.e., familiar) associates. When tested with the familiar associated pairs of stimuli, perirhinal neurons exhibited two properties that seemed directly related to the requirements for associative learning. First, neurons responded more similarly to associated stimuli (predictor and choice stimuli) than would be expected by chance. If, for example, a cell responded well to a particular predictor stimulus, then it would tend to respond well to the particular choice stimulus with which it was associated. Second, the magnitude of activity during the delay interval between predictor and choice was correlated with the responses to both the predictor and choice stimuli. In other words, delay activity was often higher on trials when the predictor and/or choice stimuli elicited good responses from the cell than when they elicited poor responses. These delay effects were largest in sessions with behavioral evidence of association learning.

By contrast, neither of these associative effects on neuronal activity was found when the stimulus pairs were novel, i.e., learned on the day of the recording. Across the population of cells, there was no correlation between the responses to associated predictor and choice stimuli on the first day. Furthermore, although the magnitude of delay activity was often correlated with the magnitude of responses to the preceding predictor stimulus on the first day, there was no correlation between the delay activity and the responses to the associated choice stimulus. In spite of this failure to find any perirhinal correlates of associative learning on the first day of learning, the monkeys' behavioral performance indicated that they did indeed learn to associate the stimuli on the first day. With novel stimuli, behavioral reaction times were faster when choice stimuli were followed by frequently associated predictors than when they were followed by infrequently associated predictors, and this was found for both invalid and neutral predictors. Thus, if we assume that the perirhinal response changes we found with familiar stimuli actually contribute to associative memory, then our failure to find these same changes with novel stimuli suggests that different mechanisms are used to perform the task on the first day than on subsequent days of training. On the first day, the critical mechanisms may be located outside of the perirhinal cortex. If so, the changes in perirhinal activity we found on the second and later days of training might be caused by a consolidation of the memory trace in perirhinal cortex after initial learning-induced activity changes in other medial temporal lobe structures. We also cannot eliminate the possibility that the critical sites of plasticity remain outside the perirhinal cortex even after extensive training and that the effects of learning we found on perirhinal responses were caused by feedback from these critical sites.

We have often found that there is little obvious perceptual similarity among the stimuli to which a given perirhinal neuron responds well and a corresponding lack of similarity among the stimuli to which a given neuron responds poorly (unpublished observations). The fact that we found that neuronal responses to temporally associated stimuli tend to become similar to one another with experience provides a possible explanation for the response properties of many perirhinal neurons. Specifically, perirhinal responses may be specific for behaviorally significant stimulus categories, rather than stimulus features per se. One could imagine, for example, that a perirhinal neuron might respond to both the image of a piece of fruit and the image of a leaf because they are often associated in time, although they share few physical features. The modification of response properties for temporally associated stimuli might also be a mechanism for learning to associate different three-dimensional views of the same object. In agreement with this, Logothetis has found that TE neurons respond more commonly to different threedimensional views of the same object than would be expected by chance (Logothetis et al., 1995).

Although the mean correlation we found between responses to familiar predictor and choice stimuli was highly significant, the actual magnitude of this correlation (0.145) was quite small across the population of cells. We estimated that $18.4 \%$ of the variance in mean firing rates of choice stimuli could be accounted for by the associated predictor stimulus. One reason why the small size of this correlation could be misleading is that associative learning might affect the responses of only a subpopulation of cells. Because we were unable to track changes in response properties over the course of daily sessions, we had no independent way to separate cells affected by learning from cells that were not. However, the increase in the number of neurons with strong positive correlations after training can be seen in the distribution of correlation coefficients for familiar stimuli.

Using a different behavioral task, Miyashita and colleagues (Sakai and Miyashita, 1991; Higuchi and Miyashita, 1996) found that the correlation between the responses to paired-associate stimuli in perirhinal cortex was similar in magnitude to what we found. They did not test for correlations in responses to novel stimuli, because all of the stimuli used in their study were highly familiar to the animal.

In contrast to the work by Miyashita and colleagues as well as our own, Sobotka and Ringo (1993) failed to observe any response similarity between paired-associate stimuli. In their study, two stimuli were presented simultaneously as a complex image, and a monkey had to discriminate GO from NO-GO stimulus pairs. It is possible that the two stimuli presented on the screen were treated by the monkey as a single complex stimulus rather than as two independent, but associated, stimuli, which might account for the failure to find any effects of learning on responses. Gochin et al. (1994) also failed to find any effects of associative learning on TE responses. In their study, which used a conditional association task with five stimuli, monkeys were trained to respond differently depending on which stimulus of a pair came first. Gochin et al. (1994) may have failed to find effects of 
association learning on responses because individual stimuli were used in more than one pair. For example, A was followed by $\mathrm{B}$ on a "GO" trial, but $\mathrm{C}$ was followed by $\mathrm{B}$ on a "NO-GO" trial. Although stimulus $\mathrm{B}$ had a different meaning depending on what preceded it, both $\mathrm{A}$ and $\mathrm{C}$ were paired with $\mathrm{B}$ an equal number of times.

Sakai and Miyashita (1991) found that delay activity was correlated with the responses to the second stimulus in a sequential paired-associate task, similar to what we found in the present study. They concluded that delay activity in perirhinal cortex is prospective in the paired-associative task. However, we found that delay activity was almost exclusively retrospective with novel stimuli in our associative task and was both retrospective and prospective with familiar stimuli. Retrospective delay activity has been found in several other studies of perirhinal and TE cortex (Li et al., 1993; Miller et al., 1993).

In a different study, Miyashita (1988) presented stimuli in a fixed temporal pattern and measured the delay activity after each stimulus. They reported that the delay activity of TE neurons was more similar after stimuli presented nearby in time compared with delay activity after stimuli not linked in time. Yakovlev et al. (1998) conducted a similar experiment and reported that the delay activity was primarily correlated with the response to the previous stimulus in the sequence. They suggested that delay activity develops and becomes longer lasting after experiencing stimuli in a fixed sequence. Neither of these two studies reported changes in stimulus-evoked responses with experience, but our results suggest a complex relationship between stimulus-evoked responses and delay activity that changes with experience. We find that delay activity is equally common with novel and familiar stimuli; however, with familiar stimuli, responses to paired stimuli become more similar, and delay activity shifts from being nearly entirely retrospective to being both retrospective and prospective.

What is the function of delay activity in the formation of associative memories? If the association is between two stimuli separated in time, then a likely function of retrospective delay activity is to maintain a representation of the first stimulus during the delay. If the neural representation of the first image is active during the presentation of the second image, this simultaneous activation of different populations could facilitate Hebbian changes in connectivity between cells in the two representations. As a result, a new stimulus class could be formed with the two associated stimuli as members. After learning takes place, prospective delay activity may serve to bias the development of a neural representation of the expected stimulus, for the sake of efficiency. Alternatively, the fact that association learning leads to a common response that starts with the presentation of the first stimulus and continues through the delay and presentation of the second stimulus suggests that the perirhinal cells serve to link visual stimuli across time, possibly representing these temporally associated stimuli as an "event."

\section{REFERENCES}

Alvarez-Royo P, Clower RP, Zola-Morgan S, Squire LR (1991) Stereotaxic lesions of the hippocampus in monkeys: determination of surgical coordinates and analysis of lesions using magnetic resonance imaging. J Neurosci Methods 38:223-232.

Crist C, Yamasaki D, Komatsu H, Wurtz R (1988) A grid system and a microsyringe for single cell recording. J Neurosci Methods 26:117-122.
Eskandar EN, Optican LM, Richmond BJ (1992) Role of inferior temporal neurons in visual memory. II. Multiplying temporal waveforms related to vision and memory. J Neurophysiol 68:1296-1306.

Fahy FL, Riches IP, Brown MW (1993) Neuronal activity related to visual recognition memory: long-term memory and the encoding of recency and familiarity information in the primate anterior and medial inferior temporal and rhinal cortex. Exp Brain Res 96:457-472.

Fuster JM, Jervey JP (1981) Inferotemporal neurons distinguish and retain behaviorally relevant features of visual stimuli. Science 212:952-955.

Fuster JM, Jervey JP (1982) Neuronal firing in the inferotemporal cortex of the monkey in a visual memory task. J Neurosci 2:361-375.

Gaffan D (1994) Dissociated effects of perirhinal cortex ablation, fornix transection and amygdalectomy: evidence for multiple memory systems in the primate temporal lobe. Exp Brain Res 99:411-422.

Gochin PM, Colombo M, Dorfman GA, Gerstein GL, Gross CG (1994) Neural ensemble coding in inferior temporal cortex. J Neurophysiol 71:2325-2337.

Gray CM, Maldonado PE, Wilson M, McNaughton BL (1995) Tetrodes markedly improve the reliability and yield of multiple single-unit isolation from multi-unit recordings in cat striate cortex. J Neurosci Methods 63:43-54.

Higuchi S, Miyashita Y (1996) Formation of mnemonic neuronal responses to visual paired associates in inferotemporal cortex is impaired by perirhinal and entorhinal lesions. Proc Natl Acad Sci USA 93:739-743.

Keppel G, Zedeck S (1989) Data analysis for research designs: analysis of variance and multiple regression/correlation approaches. New York: Freeman.

Li L, Miller EK, Desimone R (1993) The representation of stimulus familiarity in anterior inferior temporal cortex. J Neurophysiol 69:1918-1929.

Logothetis NK, Pauls J, Poggio T (1995) Shape representation in the inferior temporal cortex of monkeys. Curr Biol 5:552-563.

Lueschow A, Miller EK, Desimone R (1994) Inferior temporal mechanisms for invariant object recognition. Cereb Cortex 4:523-531.

McNaughton BL, O'Keefe J, Barnes CA (1983) The stereotrode: a new technique for simultaneous isolation of several single units in the central nervous system from multiple unit records. J Neurosci Methods 8:391-397.

Meunier M, Bachevalier J, Mishkin M, Murray EA (1993) Effects on visual recognition of combined and separate ablations of the entorhinal and perirhinal cortex in rhesus monkeys. J Neurosci 13:5418-5432.

Miller EK, Desimone R (1994) Parallel neuronal mechanisms for shortterm memory. Science 263:520-522.

Miller EK, Li L, Desimone R (1993) Activity of neurons in anterior inferior temporal cortex during a short-term memory task. J Neurosci 13:1460-1478.

Miyashita Y (1988) Neuronal correlate of visual associative long-term memory in the primate temporal cortex. Nature 335:817-820.

Murray EA, Mishkin M (1986) Visual recognition in monkeys following rhinal cortical ablations combined with either amygdalectomy or hippocampectomy. J Neurosci 6:1991-2003.

Murray EA, Gaffan D, Mishkin M (1993) Neural substrates of visual stimulus-stimulus association in rhesus monkeys. J Neurosci 13:4549-4561.

Nahm FKD, Dale AM, Albright TD, Amaral DG (1994) In vivo microelectrode localization in the brain of the alert monkey: a combined radiographic and magnetic resonance imaging approach. Exp Brain Res 98:401-411.

Naya Y, Sakai K, Miyashita Y (1996) Activity of primate inferotemporal neurons related to a sought target in pair-association task. Proc Natl Acad Sci USA 93:2664-2669.

Nichols AM, Ruffner TW, Sommer MA, Wurtz RH (1998) A screw microdrive for adjustable chronic unit recording in monkeys. J Neurosci Methods 81:185-188.

Posner MI, Snyder CR, Davidson BJ (1980) Attention and the detection of signals. J Exp Psychol 109:160-174.

Robinson DA (1963) A method of measuring eye movement using a scleral search coil in a magnetic field. IEEE Trans Biomed Eng 10:137-145.

Sakai K, Miyashita Y (1991) Neural organization for the long-term memory of paired associates. Nature 354:152-155. 
Sobotka S, Ringo JL (1993) Investigation of long-term recognition and association memory in unit responses from inferotemporal cortex. Exp Brain Res 96:28-38.

Sobotka S, Ringo JL (1996) Mnemonic responses of single units recorded from monkey inferotemporal cortex, accessed via transcommissural versus direct pathways: a dissociation between unit activity and behavior. J Neurosci 16:4222-4230.

Suzuki WA, Amaral DG (1994) Perirhinal and parahippocampal cortices of the macaque monkey: cortical afferents. J Comp Neurol 350:497-533.

Suzuki WA, Zola-Morgan S, Squire LR, Amaral DG (1993) Lesions of the perirhinal and parahippocampal cortices in the monkey produce long-lasting memory impairment in the visual and tactual modalities. J Neurosci 13:2430-2451.

Yakovlev V, Fusi S, Berman E, Zohary E (1998) Inter-trial neuronal activity in inferior temporal cortex: a putative vehicle to generate long-term visual association. Nat Neurosci 1:310-317.

Zola-Morgan S, Squire LR, Amaral DG, Suzuki WA (1989) Lesions of perirhinal and parahippocampal cortex that spare the amygdala and hippocampal formation produce severe memory impairment. J Neurosci 9:4355-4370.

Zola-Morgan S, Squire LR, Clower RP, Rempel NL (1993) Damage to the perirhinal cortex exacerbates memory impairment following lesions to the hippocampal formation. J Neurosci 13:251-265. 